\title{
Oscillatory Flow of LDL-C and Blood Fluid through a Slanted Channel with Heat within the Sight of Magnetic Field
}

\author{
K. W. Bunonyo and I. C. Eli
}

\section{ABSTRACT}

In this research, we investigated LDL-C and blood movement through a slanted channel with heat within the sight of magnetic field. In the evaluation, mathematical models for the LDL-C and blood stream and energy transfer were developed as partially coupled arrangement of partial differential equation (PDEs), the PDEs were scaled utilizing the dimensionless variables to dimensionless ordinary differential equation, they are further reduced to perturbed differential equations (ODEs) utilizing the perturbation parameters including the oscillatory term, where the non-homogenous equation and conditions are solved straightforwardly utilizing the technique for undetermined coefficient. The velocity and temperature profiles are gotten for certain overseeing boundaries included, and Mathematica codes were created utilizing simulate the impact of entering parameters on the profile. It is seen that the overseeing boundaries impacted that the entering pertinent parameters influences blood flow and it helps it controlling the LDL-C concentration, aiding treatment of atherosclerosis.

Published Online: October 11,2021

ISSN: $2684-4451$

DOI : 10.24018/ejphysics.2021.3.5.105

\section{K. W. Bunonyo*}

Department of Mathematics \& Statistics, Federal University Otuoke, Bayelsa Stat, Nigeria.

(e-mail: wilcoxbk@fuotuoke.edu.ng)

I. C. Eli

Department of Mathematics \& Statistics, Federal University Otuoke, Bayelsa Stat, Nigeria.

*Corresponding Author

Keywords: Atherosclerosis, Blood, Cardiovascular system, Heat, Hypertension, LDL-C, Magnetic Field, Metabolism, Treatment.

\section{INTRODUCTION}

Cardiovascular framework is comprised of platelets, veins and the heart. The principle capacity of the heart is to siphon blood into circulation, to the tissues and organs of the human body through the veins. As indicated by Bunonyo and Amos [1], blood as fundamental element of the essential ingredients of the body framework, and the significant constituents are red blood cells (erythrocytes), white blood cells (leukocytes), the platelet and the plasma fluid, any block to the blood stream because of the gathering of greasy substances, like cholesterol and soaked fats, inside the conduits makes an ailment called atherosclerosis or stenosis.

The presence of stenosis inside a blood vessel vein changes its stream design and haemodynamics conditions and its nonstop development inside the vein increment the odds of cardiovascular breakdown, significantly [2], [3]. As atherosclerosis is straightforwardly identified with human wellbeing, numerous scientists have explored its consequences for the blood stream thinking about different shapes and sizes. Mekheimer and Kot [4] explained utilizing Mathematical model to contemplate the math of stenosis by characterizing the shape and tightening boundaries and reasoned that impedance diminishes with expanding the upsides of the stenosis length and stenosis shape boundary, while it increments as the size of the stenosis increments.
Kamangar et al. [5] mathematically examined the consistent and transient blood stream for various mathematical states of stenosis (three-sided, curved, and trapezium) having $70 \%$ (moderate), $80 \%$ (middle of the road), and $90 \%$ (serious) stenosis. Considering an ailing vein, Tripathi and Sharma [6] utilized a Mathematical model to read restricted conduits for both center and plasma regions independently. It was shown that the speed profile of blood stream diminishes with expanding stature of the stenosis for both center and plasma locales.

Magneto-hydrodynamics (MHD) is vital in clinical science as it assists with treating hyperthermia, harmful cancer or magnetic injuries draining decrease during medical procedures [7], [8] As blood shows the qualities of bio magnetic fluids, MHD laws are utilized to consider the progression of blood through affected by magnetic field. Blood contains hemoglobin; Hemoglobin is an iron $(\mathrm{Fe})$ containing protein that transports oxygen around the body from the lungs to where it is required, similar to the cerebrum or muscles and so on During the 1930s, it was discovered that hemoglobin has magnetic properties that are diverse relying upon if it is conveying oxygen.

Over the most recent fifteen years, the distinction in magnetic property has been utilized in magnetic resonance imaging (MRI) research. X-ray utilizes an extremely impressive magnetic field so this distinction in the magnetic 
properties of oxygenated and deoxygenated hemoglobin in blood can be identified. This change is known as the BOLD (blood oxygenation level ward) signal.

Misraet al. [9] developed a Mathematical model to examine the conduct of blood course affected by outside applied magnetic field by thinking about supply route as a channel. It was seen that the speed of the blood can be controlled (increment or decline) by fluctuating the qualities of an applied magnetic force in their examination. Bhatti et al. [10] investigated the impacts of cross-over magnetic field, warmth, and mass exchange on the peristaltic movement of the two-stage stream. They tracked down that the temperature profile of the stream increments as the impact of the magnetic force increments.

In a further report, Ponalagusamy et al. [11] clarified the impacts of the magnetic field for the two-stage blood dissemination comprising of RBC containing center locale and without cell plasma area and portrayed that divider shear pressure and impedance increment as qualities of the outside applied magnetic field force increments.

In late time, the warmth produced by Joule warming and thick dispersal has grabbed the eye of numerous analysts and researchers the same.

Joule warming is the interaction wherein heat is produced because of crash among the moving particles and in this method dynamic energy is changed into heat which upgrades the temperature of the human body [12], [13] On the other hand, thick dissemination is determined through the work done by the speed against gooey anxieties and furthermore known as thick dispersal of energy [14]. The oxidation of metabolic powers, for example, carb and unsaturated fats in the mitochondria of the muscle filaments produces adenosine triphosphate. Through the hydrolysis of adenosine triphosphate, energy is delivered to help muscle withdrawal which creates heat. Be that as it may, the hydrolysis of adenosine triphosphate likewise delivers heat. In the additional examination, the fixings in the medicine, like camphor, increment blood course to the outer layer of the skin, causing a warming impression that can divert from torment and solidness. Camphor and menthol may likewise further develop blood dissemination to the muscles, possibly accelerating pending time and diminishing aggravation Bunonyo and Eli [20].

In case there is an excessive amount of LDL cholesterol in the body it can develop in different corridors, obstructing them and diminishing their adaptability. Solidifying of the corridors is results to an atherosclerosis, and that confine ordinary blood stream on account of the deficiency of the vessel adaptability, so the heart needs to work more enthusiastically to push blood through to the downstream Bunonyo et al. [15] The additional work done by the heart to push blood through because of plaque arrangement on the blood channel can prompt hypertension, and it the channel is to some extent and completely debilitated it could prompt stroke and coronary episode.

Strokes and coronary episodes, the main sources of death in the industrialized world, are regularly connected to high blood consistency. Thicker blood harms veins, and in fixing the harm, the vessels develop greasy stores, which make strokes and coronary episodes almost certain. Right now, the best way to lessen blood thickness is with drugs like anti- inflammatory medicine, which hinder the inclination of blood to cluster. Yet, ibuprofen has incidental effects: in high dosages, it can prompt stomach dying, ulcers, and even tinnitus, or ringing of the ears.

The investigation of the impact of substance response in blood stream can't be overemphasized because of its significance taking into account a few physiological and physiochemical parts of medication elements.

In most compound responses, the response rate depends on the convergence of the actual species, regardless of whether they are heterogeneous or homogeneous. Further, the compound response relies on whether it happens at an interface or as a solitary stage volume response. On the off chance that the response pace of any substance response is straightforwardly relative to the fixation it is known as a first-request homogeneous compound response. The response rate is corresponding to the nth force of the fixation is viewed as nth request synthetic response. Makinde [16] dissected the mixed convection issue for vertical permeable plate considering nth request homogeneous synthetic response between the fluid and the diffusing species.

In a further examination, Tripathi and Sharma [17] clarified the impacts of the principal request substance response on the two-stage model of blood stream and came about that the fixation profile diminishes in both center and plasma regions as upsides of the compound response boundary increment.

The principle reason for this current examination is to explore the progression of LDL-C and blood through a slanted atherosclerotic channel, propelled by the past investigates did by Bunonyo and Amos [18], Bunonyo et al. [15] and Midya [19], where they all unequivocally made it clear the different commitments of magnetic field power and warmth sources. Notwithstanding, they didn't think about the point of tendency circumstance. The point of tendency is vital because of some situation where a patient is needed to sit in a specific situation for blood dissemination to improve before a surgery should be possible. The progression of the mixed fluid, which is blood conveying blood proteins, is considered incompressible, thick and electrically leading. The administering conditions of fluid stream are addressed dependent upon the particular and applicable limit conditions. The impacts of appropriate boundaries going into the issue have been talked about exhaustively. This paper is coordinated as follows. The Mathematical definition in Section 2, arrangements of administering conditions is given in Section 3 under the technique for arrangement, mimicked results are introduced in Section 4 under show of results, conversation of results are introduced in Section 5, and Section 5 gives the ends.

\section{MATHEMATICAL FORMULATION}

In defining the stream equations to examine the mixed fluid stream in a sporadic slanted equal channel, it is considered that the fluid is a combination of LDL-C and blood, incompressible, thick and leading fluid, streaming in a slanted channel with the point of tendency, and the stream far field temperature and LDL-C focus is and, likewise the temperature and convergence of the fluid at the 
atherosclerotic region. The stream is additionally thought to be oscillatory in a permeable channel, and unidirectional with speed unidirectional with velocity $\vec{w}=\left(0,0, w^{*}\right)$ towards the $x^{*}$-axis. The height $\delta^{*}$ of irregularity is caused by stenosis, the length of the irregularity is $l_{0}^{*}$, and the flow is also assumed to be influenced by magnetic field with intensity $\vec{B}=\left(0,0, B_{0}\right)$, flowing in an inclined channel with the angle of inclination $\alpha$, and the flow far field temperature and LDL-C concentration is $T_{\infty}$ and $C_{\infty}$, also the temperature and concentration of the fluid at the atherosclerotic wall is $T_{w} \& C_{w}$. Following the previously mentioned presumptions, we present the figure showing the stream locale and the abnormality. Nonetheless, we accept that the development pace of cholesterol prompted atherosclerosis can be constrained by an inhibitor which we allude to as the treatment $R_{T}$ and the stream could be sped up by the angle of inclination. (See Fig. 1).

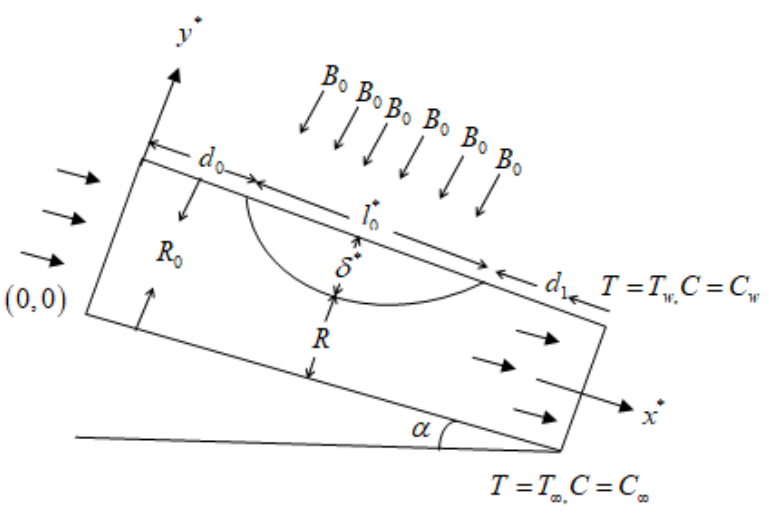

Fig. 1. Schematic diagram showing the angle of inclination and irregular region on the flow profile.

From Fig. 1, the region of atherosclerosis modeled to be:

$$
R=R_{0}-\delta^{*}\left(\cos 2 \frac{\pi x^{*}}{l_{0}^{*}}\right)
$$

\section{Continuity Equation}

$$
\frac{\partial w^{*}}{\partial x^{*}}+\frac{\partial u^{*}}{\partial y^{*}}=0
$$

\section{Momentum Equation}

$\rho_{b} \frac{\partial w^{*}}{\partial t^{*}}=-\frac{\partial P^{*}}{\partial x^{*}}+\mu_{b} \frac{\partial^{2} w^{*}}{\partial y^{* 2}}-\sigma_{e} B_{0}^{2} w^{*}-\frac{\mu_{b} \varphi}{k^{*}} w^{*}$

$+\rho_{b} g \beta_{T}\left(T^{*}-T_{\infty}\right) \cos \alpha+\rho_{b} g \beta_{C}\left(C^{*}-C_{\infty}\right) \cos \alpha$

\section{Energy Equation}

$\rho_{b} c_{p} \frac{\partial T^{*}}{\partial t^{*}}=k_{b T} \frac{\partial^{2} T^{*}}{\partial y^{* 2}}+Q_{0}\left(T^{*}-T_{\infty}\right)+Q_{1}\left(C^{*}-C_{\infty}\right)$ $\frac{\partial C^{*}}{\partial t^{*}}=D_{m} \frac{\partial^{2} C^{*}}{\partial y^{* 2}}-k_{0}\left(C^{*}-C_{\infty}\right)$

The corresponding boundary conditions are:

$$
\left.\begin{array}{ll}
w^{*}=0, T^{*}=T_{\infty}, C^{*}=C_{\infty} & \text { at } y^{*}=0 \\
w^{*}=0, T^{*}=T_{w}, C^{*}=C_{w} & \text { at } y^{*}=R
\end{array}\right\}
$$

where $k_{b T}$ is the thermal conductivity of blood, $D_{m}$ is the molecular diffusivity, $T^{*}$ is blood temperature, $\rho_{b}$ is the density of blood, $\sigma_{e}$ is electrical conductivity, $c_{b p}$ is the specific heat capacity of blood, $\beta_{T}$ is the volumetric expansion, $\beta_{C}$ is the volumetric expansion due to concentration, $\mu_{b}$ is the dynamic viscosity of blood, $\varphi$ is the porosity, $k^{*}$ is the permeability of the porous medium, $Q_{0}$ is the is the dimensional heat source and $Q_{1}$ is dimensional heat due to body metabolism.

$$
\left.\begin{array}{l}
x=\frac{x^{*}}{l_{0}^{*}}, y=\frac{y^{*}}{R_{0}}, w=\frac{w^{*} R_{0}}{v}, t=\frac{v t^{*}}{R_{0}^{2}}, R d_{1}=\frac{Q_{1} R_{0}^{2}}{k_{b T}} \frac{\left(C^{*}-C_{\infty}\right)}{\left(T_{w}-T_{\infty}\right)}, \\
\phi=\frac{C^{*}-C_{\infty}}{C_{w}-C_{\infty}}, \theta=\frac{T^{*}-T_{\infty}}{T_{w}-T_{\infty}}, P=\frac{P^{*} R_{0}^{3}}{l_{0}^{*} v \mu_{b}}, R d_{2}=\frac{Q_{0} R_{0}^{2}}{k_{b T}}, \delta=\frac{\delta^{*}}{R_{0}}
\end{array}\right\}
$$

Using the dimensionless quantities in equation (7), we reduce the equations (1) to (6) as follows:

Considering the fact that the growth of cholesterol can be controlled using inhibitor in reducing the LDL-C, we have the region of atherosclerosis in equation (1) remodeled to:

$$
\frac{R}{R_{0}}=1-\delta_{0} e^{\frac{a t}{R_{T}}}(\cos 2 \pi x)
$$

The dimensionless equations governing the flow are as follows:

$$
\frac{\partial w}{\partial t}=-\frac{\partial P}{\partial x}+\frac{\partial^{2} w}{\partial y^{2}}+G r \theta \cos \alpha+G \operatorname{cocos} \alpha-M^{2} w-\frac{w}{D a}(9)
$$

$\operatorname{Pr} \frac{\partial \theta}{\partial t}=\frac{\partial^{2} \theta}{\partial y^{2}}+R d_{2} \theta+R d_{1} \operatorname{Pr} \phi$

$\frac{\partial \phi}{\partial t}=\frac{1}{S c} \frac{\partial^{2} \phi}{\partial y^{2}}-R d_{3} \phi$

The corresponding boundary conditions are:

$\left.\begin{array}{ll}w=0, \phi=0, \theta=0 & \text { at } y=0 \\ w=0, \phi=1, \theta=1 & \text { at } y=h\end{array}\right\}$

where: 


$$
\left.\begin{array}{l}
H a=B_{0} R_{0} \sqrt{\frac{\sigma_{e}}{\mu_{b}}}, D a=\frac{k^{*}}{R_{0}^{2}}, G c=\frac{g \beta_{C}\left(C_{w}-C_{\infty}\right) R_{0}^{3}}{v^{2}}, \\
G r_{1}=G r h \cos \alpha, S c=\frac{v}{D_{m}}, G r=\frac{g \beta_{T}\left(T_{w}-T_{\infty}\right) R_{0}^{3}}{v^{2}}, \\
\operatorname{Pr}=\frac{\mu_{b} c_{p}}{k_{b T}}, G c_{1}=G \operatorname{chcos} \alpha
\end{array}\right\}
$$

\section{Method OF SOlution}

Since the flow is purely oscillatory due to the pumping action of the ventricle, it is appropriate to seek oscillatory solutions in the form:

$$
\left.\begin{array}{l}
w(y, t)=w_{0}(y) e^{i \omega t} \\
\theta(y, t)=\theta_{0}(y) e^{i \omega t} \\
\phi(y, t)=\phi_{0}(y) e^{i \omega t} \\
-\frac{\partial P}{\partial x}=P_{0} e^{i \omega t}, \chi=\frac{y}{h}
\end{array}\right\}
$$

Using the solution form in equation (13), we reduce the dimensionless coupled partial differential equation (9)-(12a) to homogenous and non-homogenous ordinary differential equations in the form:

$$
\begin{aligned}
& \frac{\partial^{2} w_{0}}{\partial \chi^{2}}-h\left(i \omega+M^{2}+\frac{1}{D a}\right) w_{0}=P_{0} h-G r_{1} \theta_{0}-G c_{1} \phi_{0} \\
& \frac{\partial^{2} \theta_{0}}{\partial \chi^{2}}+h\left(R d_{2}-i \omega\right) \operatorname{Pr} \theta_{0}=-R d_{1} h \phi_{0} \\
& \frac{\partial^{2} \phi_{0}}{\partial \chi^{2}}-h\left(R d_{3}+i \omega\right) S c \phi_{0}=0
\end{aligned}
$$

Subject to the following boundary conditions as:

$$
\left.\begin{array}{ll}
w_{0}=0, \phi_{0}=0, \theta_{0}=0 & \text { at } \chi=0 \\
w_{0}=0, \phi_{0}=e^{-i \omega t}, \theta_{0}=e^{-i \omega t} & \text { at } \chi=1
\end{array}\right\}
$$

Solving for the LDL-C concentration Equation (3.15), we have:

$$
\phi_{0}(\chi)=A_{1} \sinh \left(\sqrt{\beta_{3}} \chi\right)+B_{1} \cosh \left(\sqrt{\beta_{3}} \chi\right)
$$

where $\beta_{3}=h\left(R d_{3}+i \omega\right) S c$.

Solving for the constant coefficients in Equation (18) using the appropriate boundary condition in Equation (17), we have:

$$
\phi_{0}(\chi)=\frac{e^{-i \omega t}}{\sinh \left(\sqrt{\beta_{3}}\right)} \sinh \left(\sqrt{\beta_{3}} \chi\right)
$$

Substitute Equation (19) into perturbed energy equation (15), we have:

$\frac{\partial^{2} \theta_{0}}{\partial \chi^{2}}+h\left(R d_{2}-i \omega\right) \operatorname{Pr} \theta_{0}=-\frac{R d_{1} h e^{-i \omega t}}{\sinh \left(\sqrt{\beta_{3}}\right)} \sinh \left(\sqrt{\beta_{3}} \chi\right)$

Let $\beta_{4}=-\frac{R d_{1} h e^{-i \omega t}}{\sinh \left(\sqrt{\beta_{3}}\right)}$, so that equation (20) reduces to:

$\frac{\partial^{2} \theta_{0}}{\partial \chi^{2}}+\beta_{2} \theta_{0}=\left(\beta_{4} \sinh \left(\sqrt{\beta_{3}} \chi\right)\right)$

where $\beta_{2}=h\left(R d_{2}-i \omega\right) \operatorname{Pr}$.

Solution of the homogenous part of equation (21) is:

$\theta_{0 h}(\chi)=A_{2} \sin \left(\sqrt{\beta_{2}} \chi\right)+B_{2} \cos \left(\sqrt{\beta_{2}} \chi\right)$

and the solution of the particular part of equation (21) is:

$\theta_{0 p}=\frac{\beta_{4}}{\left(\beta_{3}+\beta_{2}\right)} \sinh \left(\sqrt{\beta_{3}} \chi\right)$

So that, the general solution of equation (21) would be:

$\theta_{0}(\chi)=A_{2} \sin \left(\sqrt{\beta_{2}} \chi\right)+\frac{\beta_{4}}{\left(\beta_{3}+\beta_{2}\right)} \sinh \left(\sqrt{\beta_{3}} \chi\right)$

where $B_{2}=0, A_{2}=\frac{e^{-i \omega t}}{\sin \left(\sqrt{\beta_{2}}\right)}-\frac{\beta_{4}}{\left(\beta_{3}+\beta_{2}\right)} \frac{\sinh \left(\sqrt{\beta_{3}}\right)}{\sin \left(\sqrt{\beta_{2}}\right)}$

Substitute the LDL-C concentration equation (19) and temperature profile equation (24) in the perturbed momentum equation (14), we have:

$\frac{\partial^{2} w_{0}}{\partial \chi^{2}}-h\left(i \omega+M^{2}+\frac{1}{k}\right) w_{0}=P_{0} h-$
$\left(A_{2} G r_{1} \sin \left(\sqrt{\beta_{2}} \chi\right)+\frac{\beta_{4} G r_{1}}{\left(\beta_{3}+\beta_{2}\right)} \sinh \left(\sqrt{\beta_{3}} \chi\right)\right)$

$-\left(\frac{G c_{1} e^{-i \omega t}}{\sinh \left(\sqrt{\beta_{3}}\right)} \sinh \left(\sqrt{\beta_{3}} \chi\right)\right)$

Further simplification of equation (25) reduces to:

$\frac{\partial^{2} w_{0}}{\partial \chi^{2}}-\left(i \omega+M^{2}+\frac{1}{k}\right) w_{0}=$

$\left(\begin{array}{l}P_{0} h-A_{2} G r_{1} \sin \left(\sqrt{\beta_{2}} \chi\right) \\ -\left(\frac{G c_{1} e^{-i \omega t}}{\sinh \left(\sqrt{\beta_{3}} h\right)}+\frac{\beta_{4} G r_{1}}{\left(\beta_{3}+\beta_{2}\right)}\right) \sinh \left(\sqrt{\beta_{3}} \chi\right)\end{array}\right)$ 
Let $\beta_{1}=h\left(i \omega+M^{2}+\frac{1}{D a}\right)$,

$\beta_{5}=\left(\frac{G c_{1} e^{-i \omega t}}{\sinh \left(\sqrt{\beta_{3}} h\right)}+\frac{\beta_{4} G r_{1}}{\left(\beta_{3}+\beta_{2}\right)}\right)$ so that equation (26) is

reduce to:

$$
\begin{aligned}
& \frac{\partial^{2} w_{0}}{\partial \chi^{2}}-\beta_{1} w_{0}=P_{0} h-A_{2} G r_{1} \sin \left(\sqrt{\beta_{2}} \chi\right) \\
& -\beta_{5} \sinh \left(\sqrt{\beta_{3}} \chi\right)
\end{aligned}
$$

If the RHS of equation (27) is zero, the solution is:

$w_{0 h}(\chi)=A_{6} \sinh \left(\sqrt{\beta_{1}} \chi\right)+B_{6} \cosh \left(\sqrt{\beta_{1}} \chi\right)$

If the RHS ofequation (27) is not zero, the particular solution will be of the form:

$w_{0 p}=A_{31}+A_{4} \sin \left(\sqrt{\beta_{2}} \chi\right)+A_{5} \sinh \left(\sqrt{\beta_{3}} \chi\right)$

where

$$
A_{31}=-\frac{P_{0} h}{\beta_{1}}, A_{4}=\frac{A_{2} G r_{1}}{\left(\beta_{2}+\beta_{1}\right)}, A_{5}=\frac{\beta_{5}}{\left(\beta_{1}-\beta_{3}\right)}, B_{4}=0, B_{5}=0
$$

Then, the general solution to equation (27) is:

$$
w_{0}(\chi)=\left(\begin{array}{l}
A_{6} \sinh \left(\sqrt{\beta_{1}} \chi\right)+B_{6} \cosh \left(\sqrt{\beta_{1}} \chi\right) \\
+A_{31}+A_{4} \sin \left(\sqrt{\beta_{2}} \chi\right)+A_{5} \sinh \left(\sqrt{\beta_{3}} \chi\right)
\end{array}\right)
$$

We use the boundary conditions in equation (17) to solve for the coefficients in equation (30) so that the solution becomes:

$$
\begin{aligned}
& w_{0}(\chi)=A_{6} \sinh \left(\sqrt{\beta_{1}} \chi\right)-A_{31}\left(\cosh \left(\sqrt{\beta_{1}} \chi\right)-1\right) \\
& +A_{4} \sin \left(\sqrt{\beta_{2}} \chi\right)+A_{5} \sinh \left(\sqrt{\beta_{3}} \chi\right)
\end{aligned}
$$

where:

$$
\begin{aligned}
& A_{6}=A_{31}\left(\frac{\cosh \left(\sqrt{\beta_{1}}\right)}{\sinh \left(\sqrt{\beta_{1}}\right)}-\frac{1}{\sinh \left(\sqrt{\beta_{1}}\right)}\right) \\
& -A_{4} \frac{\sin \left(\sqrt{\beta_{2}}\right)}{\sinh \left(\sqrt{\beta_{1}}\right)}-A_{5} \frac{\sinh \left(\sqrt{\beta_{3}}\right)}{\sinh \left(\sqrt{\beta_{1}}\right)}
\end{aligned}
$$

We can now substitute equation (31), (24) and (19) into equation (13) in order to obtain our blood velocity profile, temperature profile and LDL-C profile respectively as:

$$
w(\chi, t)=\left(\begin{array}{l}
A_{6} \sinh \left(\sqrt{\beta_{1}} \chi\right)-A_{31}\left(\cosh \left(\sqrt{\beta_{1}} \chi\right)-1\right) \\
+A_{4} \sin \left(\sqrt{\beta_{2}} \chi\right)+A_{5} \sinh \left(\sqrt{\beta_{3}} \chi\right)
\end{array}\right) e^{i \omega t}
$$$$
\theta(\chi, t)=\left(\begin{array}{l}
A_{2} \sin \left(\sqrt{\beta_{2}} \chi\right)+B_{2} \cos \left(\sqrt{\beta_{2}} \chi\right) \\
+\frac{\beta_{4}}{\left(\beta_{3}+\beta_{2}\right)} \sinh \left(\sqrt{\beta_{3}} \chi\right)
\end{array}\right) e^{i \omega t}
$$$$
\phi(\chi, t)=\left(\frac{e^{-i \omega t}}{\sinh \left(\sqrt{\beta_{3}}\right)} \sinh \left(\sqrt{\beta_{3}} \chi\right)\right) e^{i \omega t}
$$

The volumetric flow rate is solved analytically using equation (32), which is

$$
Q=\int_{0}^{1}\left(\begin{array}{l}
A_{6} \sinh \left(\sqrt{\beta_{1}} \chi\right)-A_{31}\left(\cosh \left(\sqrt{\beta_{1}} \chi\right)-1\right) \\
+A_{4} \sin \left(\sqrt{\beta_{2}} \chi\right)+A_{5} \sinh \left(\sqrt{\beta_{3}} \chi\right)
\end{array}\right) e^{i \omega t} d \chi
$$

$$
Q=e^{i \omega t}\left(\begin{array}{l}
\frac{A_{6}}{\sqrt{\beta_{1}}} \cosh \left(\sqrt{\beta_{1}}\right)-A_{31}\left(\frac{\sinh \left(\sqrt{\beta_{1}}\right)}{\sqrt{\beta_{1}}}-1\right) \\
-\frac{A_{4}}{\sqrt{\beta_{2}}} \cos \left(\sqrt{\beta_{2}}\right)+\frac{A_{5}}{\sqrt{\beta_{3}}} \cosh \left(\sqrt{\beta_{3}}\right) \\
-\left(\frac{A_{6}}{\sqrt{\beta_{1}}}-\frac{A_{4}}{\sqrt{\beta_{2}}}+\frac{A_{5}}{\sqrt{\beta_{3}}}\right)
\end{array}\right)
$$

The rate of heat transfer at the wall of the vessel is calculated analytically as:

$$
N u=h\left(\begin{array}{l}
B_{2} \sqrt{\beta_{2}} \sin \left(\sqrt{\beta_{2}}\right) \\
-A_{2} \sqrt{\beta_{2}} \cos \left(\sqrt{\beta_{2}}\right)-\frac{\beta_{4} \sqrt{\beta_{3}}}{\left(\beta_{3}+\beta_{2}\right)} \cosh \left(\sqrt{\beta_{3}}\right)
\end{array}\right) e^{i \omega t}(37)
$$

The rate of LDL-C mass transfer at the wall of the vessel is calculated analytically as:

$$
S h=-\left.\frac{\partial \phi}{\partial \chi}\right|_{\chi=1}=-h\left(\frac{\sqrt{\beta_{3}} e^{-i \omega t}}{\sinh \left(\sqrt{\beta_{3}}\right)} \cosh \left(\sqrt{\beta_{3}}\right)\right) e^{i \omega t}
$$

\section{Presentation of Results}

This research investigated the oscillatory flow of LDL-C and blood fluid through an inclined channel with heat in the presence of magnetic field, and the object of study has been achieved with the simulated results showing the effect of the various pertinent parameters values such as radiation, metabolic heat, Prandtl number, Hartmann number, Schmidt number, angle of inclination, chemical reaction, oscillatory frequency parameter, height of stenosis, treatment 
parameter, growth rate of $a=0.05$ at $x=0.8$ on the axial blood velocity, LDL-C concentration and temperature profiles respectively with the aid of Mathematica, version 10 .

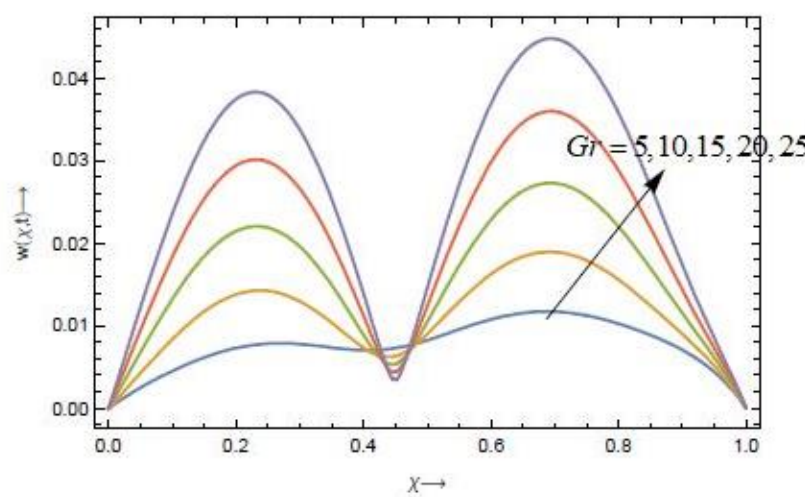

Fig. 2. Effect of Grashof parameter $G r$ values on Velocity Profile.

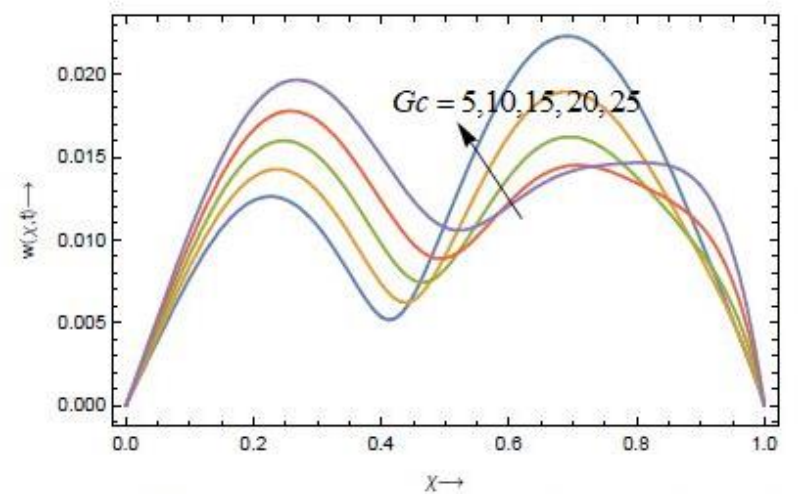

Fig. 3. Effect of solutalGrashof parameter $G c$ values on Velocity Profile.

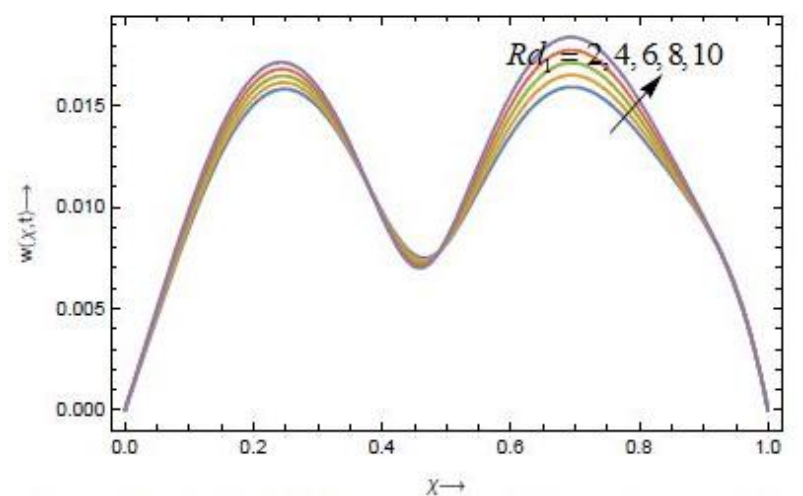

Fig. 4. Effect of Radiation parameter $R d_{l}$ values on Velocity Profile.

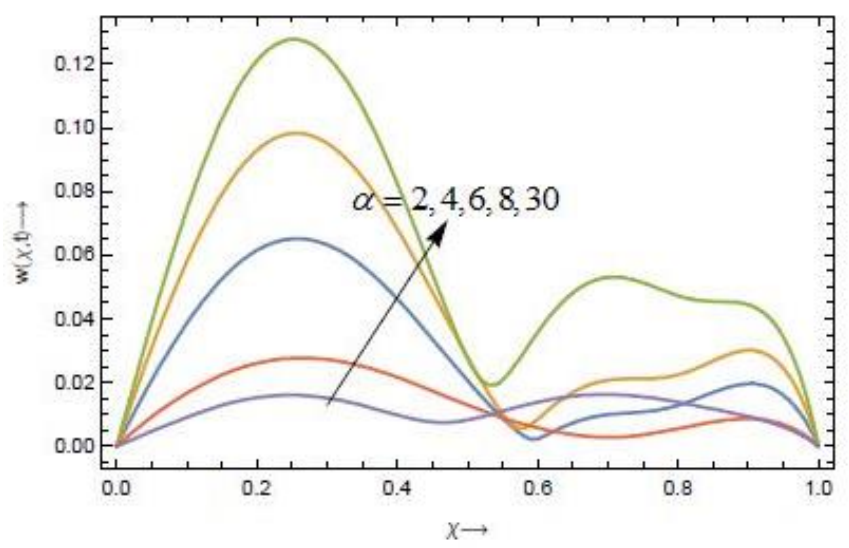

Fig. 5. Effect of inclined angle $\alpha$ values on Velocity Profile.

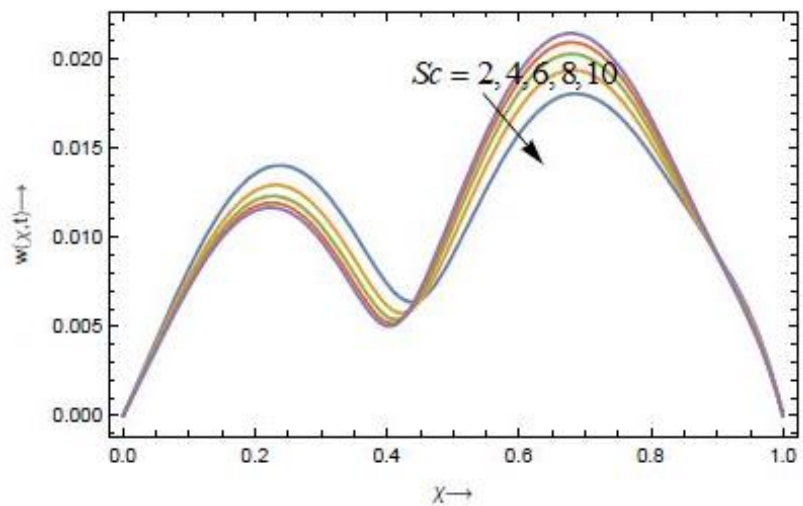

Fig. 6. Effect of Schmidt number $S c$ values on Velocity Profile.

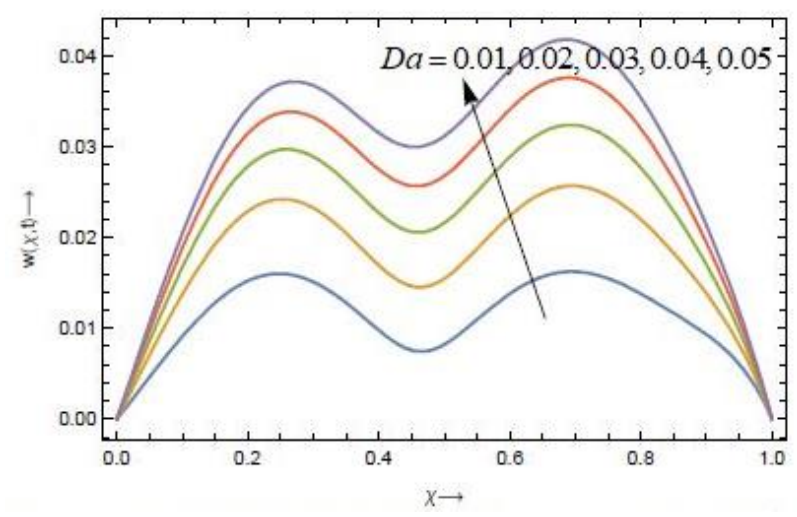

Fig. 7. Effect of Darcy parameter $D a$ values on Velocity Profile.

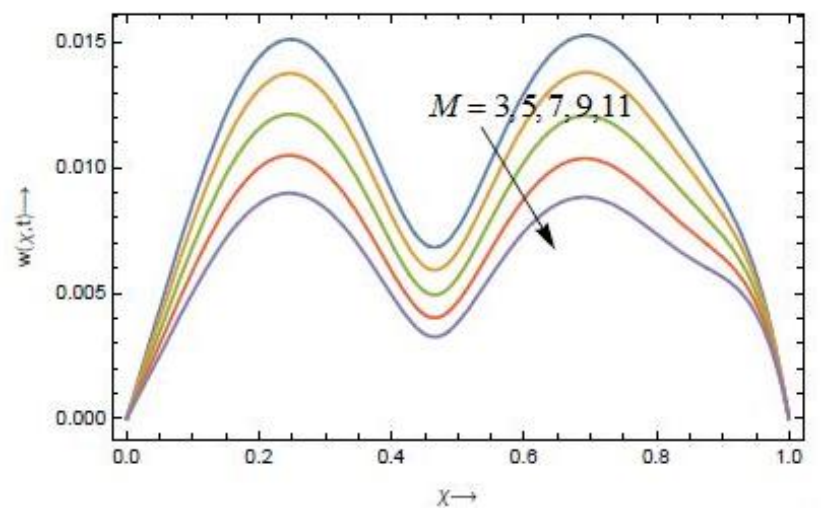

Fig. 8. Effect of Magnetic parameter $M$ values on Velocity Profile.

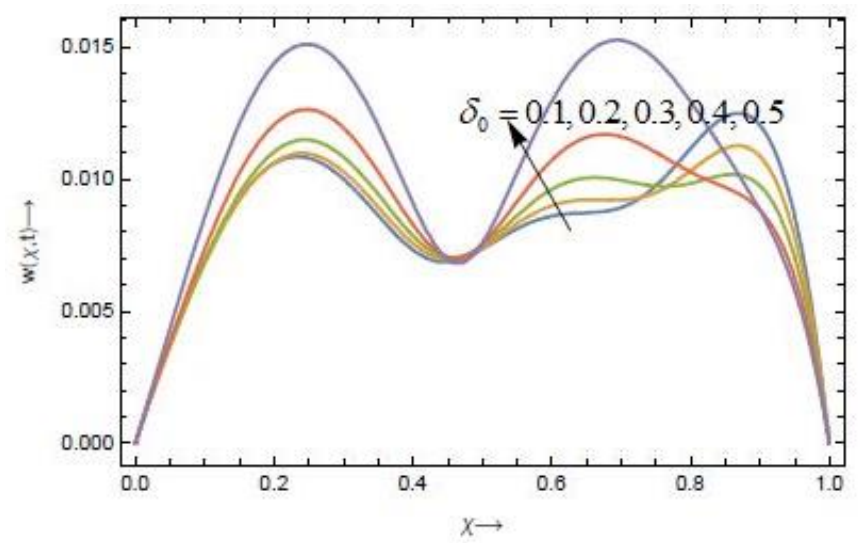

Fig. 9. Effect of Height of Stenosis $\delta_{0}$ values on Velocity Profile. 


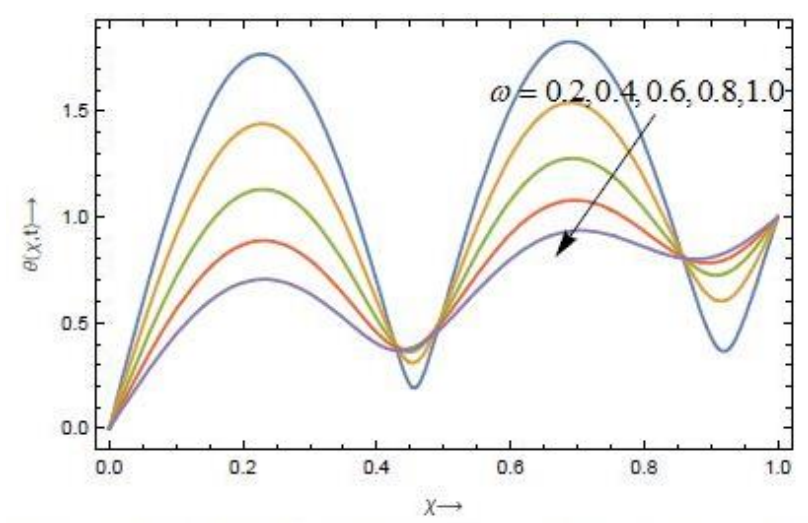

Fig. 10. Effect of Oscillatory frequency $\omega$ values on Temperature Profile.

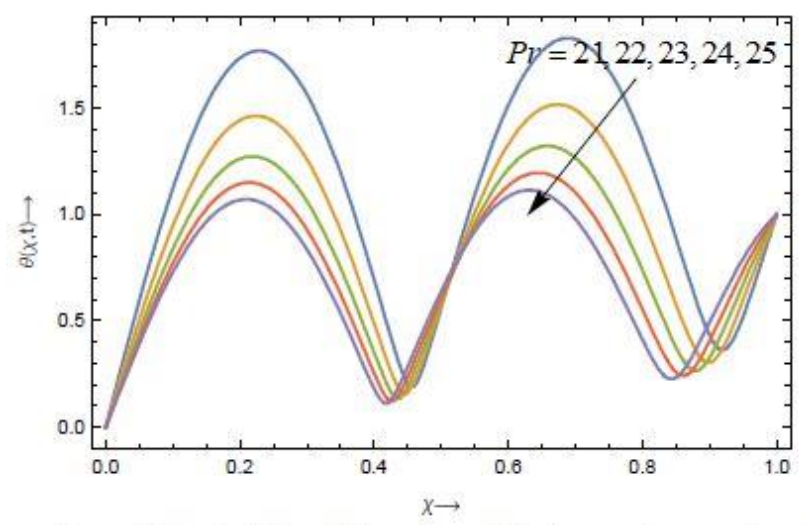

Fig. 11. Effect of Prandtl number $\operatorname{Pr}$ values on Temperature Profile.

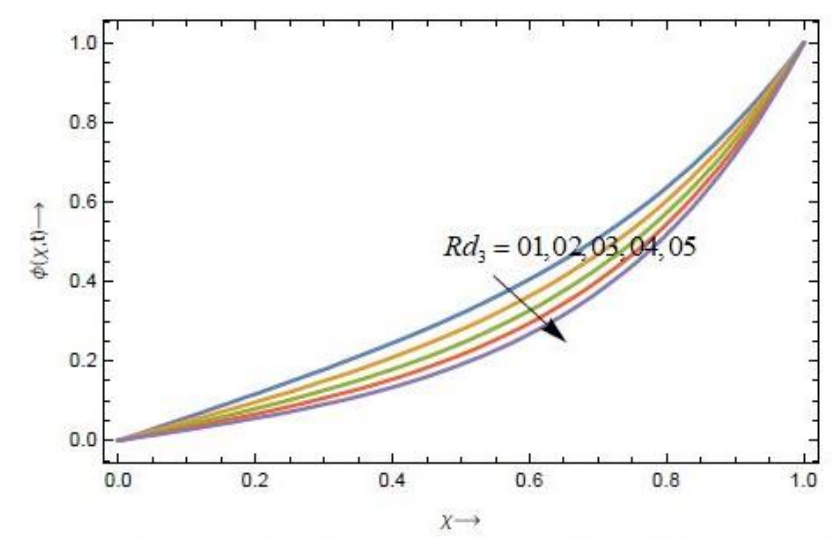

Fig. 12. Effect of Schmidt number $S c$ values on LDL-C concentration.

\section{DISCUSSION OF RESUlTS AND CONCLUSION}

The biological models led to an analytical solution of the velocity, LDL-C concentration and temperature profiles, simulation was done to investigate the effect of the entering pertinent parameters where results were presented, and the results are discussed below:

1. The increase in Grashof number $G r$ and solutal Grashof number $G c$ caused the velocity of the fluid to increase. This result shows that the velocity of the fluid increase as the buoyancy effect due to temperature and concentration lipid is increased as noticed in Fig. 2, 3. This result depicts situation whereby other pertinent parameters $G r=10, R d_{1}=03, R d_{2}=03$,

such as $R d_{3}=03, \alpha=30, \operatorname{Pr}=21, \quad \delta_{0}=0.5, R_{T}=0.5$.

$$
D a=0.01, M=0.3, \omega=0.2,
$$

2. Fig. 4 shows that the velocity increases, for an increase for different values of the metabolic heat parameter, while other parameters are kept constant. The effect of an angle of inclination was also investigated and the simulation found that the velocity profile increases for different values of angle of inclination, and the result is shown in Fig. 5. Fig. 6 illustrates a decrease in velocity profile for different values of the Schmidt number, this result is of the view that the increase in molecular diffusion could create an increase in lipid concentration in the bloodstream and that caused the velocity decrease. However, in Fig. 7, the velocity profile of the fluid increases, for different values of Darcy number. The Darcy number has to do with increase in porosity of the fluid medium, which shows that the increase in the sizes of the pore matrix of the medium the faster the flow become.

3 . The interaction between a moving electrically conducting fluid and a magnetic field would lead to a force called the Lorentz force which inhibits the velocity of the fluid as depicted by Fig. 8 .

4. Ordinarily the increase in height of stenosis would have cause a decrease in velocity profile but Fig. 9 shows otherwise because the inhibitor in the form of statin drug has been able to block off the production of cholesterol into circulation and mob up process in the form of macrophages which helps in improving the velocity profile of the fluid. Fig. 10 depicts the periodic increase in pulse rate and that led to decrease in temperature profile of the fluid, for an increase in values of the oscillatory frequency.

5. The temperature profile is seen to decrease for different values of Prandtl number, in a similar vein, the chemical reaction increases decreases the concentration of LDL-C concentration in an inclined system as shown in Fig 11,12 .

In conclusion, we have done justice to the matter in the sense that, we formulated the model and soled to and obtained an analytical solution for the flow expression, developed numerical simulation code using Mathematica, and simulated the various expression for different entering parameters in the velocity profile, temperature profile and LDL-C concentration profiles respectively. And found the significant flow parameters affecting the profiles and if well managed are of good information for health practitioners for studying cardiovascular ailments.

\section{REFERENCES}

[1] KW. Bunonyo, E. Amos. Lipid Concentration Effect on Blood Flow Through an Inclined Arterial Channel with Magnetic Field. Mathematical Modelling and Applications, 5(3); pp. 129, 2020.

[2] CJ. Cooper, TP. Murphy, DE. Cutlip, K. Jamerson, W. Henrich, DM Reid, DJ. Cohen, AH. Matsumoto, M. Steffes, MR. Jaff, MR. Prince. Stenting and medical therapy for atherosclerotic renal-artery stenosis. New England Journal of Medicine, 370(1); pp.13-22, 2014.

[3] WJ. Jean, I. Al-Bitar, DL. Zwicke, SC. Port, DH. Schmidt, TK Bajwa, High incidence of renal artery stenosis in patients with coronary artery disease. Catheterization and cardiovascular diagnosis, 32(1), pp. 8-10, 1994.

[4] KS. Mekheimer, MA. El Kot. The micropolar fluid model for blood flow through a tapered artery with a stenosis. Acta Mechanica Sinica, 24(6), pp.637-44, 2008.

[5] S. Kamangar, NJ Salman, IA. Badruddin, N. Al-Rawahi, A. Husain, K. Govindaraju, Y. Khan. Effect of stenosis on hemodynamics in left coronary artery based on patient-specific CT scan. Biomedical materials and engineering, 30(4), pp.463-73, 2019.

[6] B. Tripathi, BK. Sharma. Influence of heat and mass transfer on MHD two-phase blood flow with radiation. In AIP Conference Proceedings 1975(1), 2018 
[7] MM. Bhatti, A. Zeeshan. Study of variable magnetic field and endoscope on peristaltic blood flow of particle-fluid suspension through an annulus. Biomedical Engineering Letters, 6(4), pp. 242-9, 2016.

[8] K. Buckenmaier, A. Pedersen, P. SanGiorgio, K. Scheffler, J. Clarke, B. Inglis. Feasibility of functional MRI at ultralow magnetic field via changes in cerebral blood volume. NeuroImage, 186, pp.185-91, 2019.

[9] JC. Misra, A. Sinha, GC. Shit. A numerical model for the magnetohydrodynamic flow of blood in a porous channel. Journal of Mechanics in Medicine and Biology, 11(03), pp. 547-62, 2011.

[10] MM. Bhatti, A. Zeeshan, R. Ellahi, N. Ijaz. Heat and mass transfer of two-phase flow with Electric double layer effects induced due to peristaltic propulsion in the presence of transverse magnetic field. Journal of Molecular Liquids, 230, pp.237-46, 2017.

[11] R. Ponalagusamy, RT. Selvi. Blood flow in stenosed arteries with radially variable viscosity, peripheral plasma layer thickness and magnetic field. Meccanica. 2013; 48(10):2427-38.

[12] T. Hayat, S. Ayub, A. Tanveer, A. Alsaedi. Slip and Joule heating effects on peristaltic transport in an inclined channel. Journal of Thermal Science and Engineering Applications, 10(3), 2018.

[13] BK. Sharma, A. Mishra, S. Gupta. Heat and mass transfer in magnetobiofluid flow through a non-Darcian porous medium with Joule effect. Journal of Engineering Physics and Thermophysics, 86(4), pp. 766-74, 2013.

[14] M. Sheikholeslami, SA. Shehzad. Simulation of water based nanofluid convective flow inside a porous enclosure via nonequilibrium model. International Journal of Heat and Mass Transfer, 20, pp.1200-12, 2018.

[15] KW. Bunonyo, C. Israel-Cookey, and E. Amos. "Modeling of blood flow through stenosed artery with heat in the presence of magnetic field." Asian Research Journal of Mathematics, 6(1), pp.1-14, 2018.

[16] OD. Makinde. MHD mixed-convection interaction with thermal radiation and nth order chemical reaction past a vertical porous plate embedded in a porous medium. Chemical Engineering Communications, 198(4), pp. 590-608, 2010.

[17] B. Tripathi, BK. Sharma. Influence of Heat and Mass Transfer on Two-Phase Blood Flow with Joule Heating and Variable Viscosity in the Presence of Variable Magnetic Field. International Journal of Computational Methods, 17(03), pp.1850139, 2020.

[18] KW. Bunonyo, E. Amos. Impact of Treatment Parameter on Blood Flow in an Atherosclerotic Artery. American Journal of Theoretical and Applied Statistics. 2020; 9(3):74.

[19] C. Midya, GC. Layek, AS. Gupta, TR. Mahapatra. Magnetohydrodynamic viscous flow separation in a channel with constrictions. J. Fluids Eng, 125(6), pp. 52-62, 2003.

[20] KW. Bunonyo, IC. Eli. Mathematical Modeling of an Oscillatory MHD Blood Flow through a Lipid Saturated Porous Channel with Metabolic Heat and Magnetic Field. Communication in Physical Sciences, 6(1), pp.50, 2020.

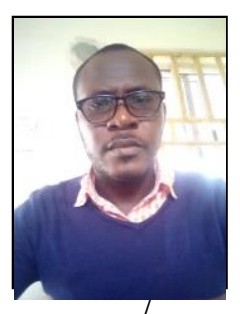

K.W. Bunonyo, hail from Bayelsa State of Nigeria, he was born in Nigeria. He studied Applied Mathematics at the post graduate level at the Rivers State University, Port Harcourt, with research interest in Haemodynamics, MHD and Mathematical Modeling, Applied Mathematics and Biostatistics. $\mathrm{He}$ is a Lecturer at the department of Mathematics and Statistics, Federal University Otuoke, Bayelsa State. 